\title{
What are the greatest challenges or barriers to applying post-mortem imaging in pediatric radiology?
}

\author{
Gary M. Hatch
}

Received: 29 June 2014 / Revised: 15 July 2014 / Accepted: 6 August 2014 / Published online: 23 September 2014

(C) Springer-Verlag Berlin Heidelberg 2014

Postmortem radiography is well-established in pediatric medico-legal death investigation, but several barriers impede the incorporation of advanced imaging:

1 Perception. The perceived lack of access to advanced imaging is exaggerated, especially in the United States, where $\mathrm{CT}$ and MR are ubiquitous. Many scanners sit idle during evening and overnight hours; if staff were made available through the night, several more scans could be performed without inconveniencing patients or changing waiting times. Access merely requires forensic practitioners to reach out to their local radiology counterparts (or vice versa).
2 Habit. Many processes in medico-legal death investigation in the United States are not evidence-based; rather they proceed according to individual training or because "that is how it's always been done." Even if uniform guidelines were established, they would be difficult to enforce if practitioners resisted changing their habits.

3 Comfort. Forensic practitioners train and practice without advanced imaging. Embracing advanced imaging requires leaving one's comfort zone, relinquishing some level of control and placing trust in new modalities and new colleagues.

Conflicts of interest None
G. M. Hatch

Radiology-Pathology Center for Forensic Imaging,

Departments of Radiology and Pathology,

University of New Mexico School of Medicine,

Albuquerque, NM, USA

\section{G. M. Hatch $(\bowtie)$}

Office of the Medical Investigator, University of New Mexico,

1101 Camino de Salud NE MSC 07 4040,

Albuquerque, NM 87131-0001, USA

e-mail: ghatch@salud.unm.edu 